\title{
Design Analysis of 1550-nm GaAsSb-(In)GaAsN Type-II Quantum-Well Laser Active Regions
}

\author{
Nelson Tansu and Luke J. Mawst, Senior Member, IEEE
}

\begin{abstract}
A novel active region design is proposed to achieve long-wavelength $(\lambda=1550$-nm) diode lasers based on a type-II quantum-well ( $Q$ W) design of (In)GaAsN-GaAsSb grown on a GaAs substrate. The strain-compensated structures hold potential as an ideal active region for $1500-\mathrm{nm}$ GaAs-based vertical cavity surface emitting lasers. A design analysis and optimization of 1550-nm emitting structures is presented. An optimal type-II multiple-QW design allows for electron-hole wavefunction overlaps of greater than $50 \%$.
\end{abstract}

Index Terms-Diode lasers, epitaxial growth, GaAsN quantum well, GaAsSb quantum well, InGaAsN quantum well, long-wavelength lasers, strain, type-II quantum-well lasers, vertical cavity lasers.

\section{INTRODUCTION}

$\mathbf{I}$ nP-BASED vertical cavity surface emitting lasers (VCSELs) emitting in the $1.55-\mu \mathrm{m}$ wavelength region have been developed based on the technologies of wafer-bonding distributed Bragg reflectors (DBRs) [1], metamorphic DBRs [2], Sb-based DBRs [3], dielectric DBRs [4], and air/semiconductor DBRs [5]. Due to the sophisticated and challenging fabrication processes involved, the lasing performance of InP-based VCSELs are typically inferior to those of GaAs-based (shorter wavelength) VCSELs and InP-based edge-emitting lasers. The conventional active region for achieving $1.55-\mu \mathrm{m}$ wavelength operation is based on InGaAs- or InGaAsP-multiple quantum wells (MQWs) on an InP-substrate [6]. Unfortunately, these lasers are highly temperature sensitive [6], due to several factors including Auger recombination, carrier leakage processes, intervalence band absorption (IVBA), and a strong temperature dependence of the material gain. The temperature-sensitive active region and the difficulty in forming high-quality DBRs on InP substrates has led to the exploration of alternate active layer materials to achieve 1550-nm emission using GaAs substrates.

Although high-performance InGaAsN-active quantum well (QW) lasers using GaAs substrates have been realized with emission wavelengths in the range of 1300-1380 nm [7]-[9], high-performance 1550-nm InGaAsN QW lasers are still difficult to realize with low threshold current density [10]. Aside from the efforts in InGaAsN technology, there have

Manuscript received May 6, 2003; revised June 24, 2003.

N. Tansu is with the Center for Optical Technologies, Department of Electrical and Computer Engineering, Lehigh University, Bethlehem, PA 18015 USA (e-mail: Tansu@Lehigh.Edu).

L. J. Mawst is with the Reed Center for Photonics, Department of Electrical and Computer Engineering, University of Wisconsin-Madison, Madison, WI 53706 USA.

Digital Object Identifier 10.1109/JQE.2003.817235 been several efforts in extending the emission wavelength on GaAs substrates utilizing highly strained $\operatorname{InGaAsN}(\mathrm{Sb})$ QWs [11] and InGaAs-GaAsSb type-II QWs [12]. While preliminary results are encouraging, the $\operatorname{InGaAsN}(\mathrm{Sb}) \mathrm{QW}$ and the InGaAs-GaAsSb type-II QW lasers are still in an early stage of development, making it difficult ascertain their potential for high-performance in the 1550-nm wavelength regime. Furthermore, extending the wavelengths beyond 1.55 $\mu \mathrm{m}$ with these technologies is very challenging, due to the requirement for an excessively large strain of the QW. New active layer materials are needed to achieve longer-wavelength device performances surpassing those of conventional InP- and GaSb-based lasers.

Here, we propose an alternative method to achieve $1.55-\mu \mathrm{m}$ emission on a GaAs substrate, based on a novel design consisting of (In)GaAsN-GaAsSb type-II multiple-quantum-wells (MQWs) utilizing the modified "W"-shape transition [12], [13]. The proposed structure has significant advantages over previous type-II QW structures on GaAs substrates [12], including built-in strain-compensation, strong carrier-confinement, and large electron-hole overlap. A preliminary design analysis indicates that a MQW structure can provide electron-hole wavefunction overlaps of greater than $50 \%$ for structures emitting at $1500-\mathrm{nm}$, significantly higher than previously reported $1300-1500 \mathrm{~nm}$ type-II QW designs (37.6\% for 1357-nm emitting structures, see [12]). The proposed design has strong potential for realizing high-performance monolithic GaAs-based VCSELs in the $1.55-\mu \mathrm{m}$ wavelength regime.

\section{Design OF “ZIG-ZAG” GaAsN-GaAsSB TyPE-II QW}

A schematic energy band diagram of the so-called "zig-zag" design, as shown in Fig. 1, is based on the modified W-shape transition [12], [13]. The electron wells consist of GaAsN layers and the hole wells consist of GaAsSb layers. The arrangement of this design is made possible due to the large disparity of the band lineup of the GaAsN and GaAsSb compounds with that of GaAs. As shown by Wu et al. [14], GaAsN has a weak type-II band lineup with a very small negative valence band offset $\left(\Delta \mathrm{E}_{\mathrm{v}}\right)$ of $20 \mathrm{meV} / \% \mathrm{~N}$. The weak type-II band lineup near the valence band of the GaAsN-GaAs, results in a large conduction band offset $\left(\Delta \mathrm{E}_{\mathrm{c}}\right)$. We employ the model solid theory [15], which has been previously employed by several groups [12], [13], [16] in determining the band edge of the GaAsSb. As shown by Liu et al. [16], GaAsSb-GaAs has a large type-II band lineup in the conduction band, which results in the large $\Delta \mathrm{E}_{\mathrm{v}}$. 


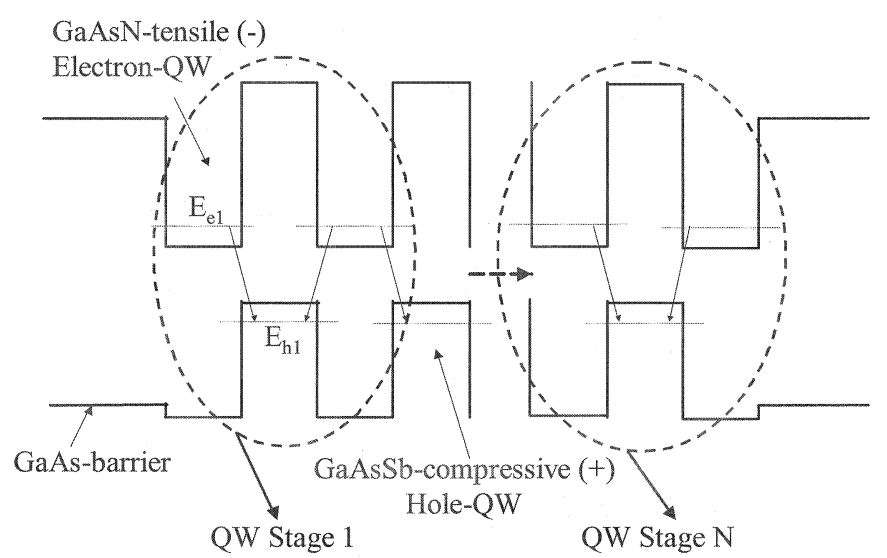

Fig. 1. Schematic energy band diagram of "zig-zag" GaAsN-GaAsSb type-II QWs with multiple stages.

Indium may also be added to the GaAsN layers to optimize the design. The potential advantages of the InGaAsN electron-QW is the smaller electron effective mass for the InGaAsN QW in comparison to that of GaAsN, allowing for increased spreading of the electron wavefunction (i.e., improved electron-hole wavefunction overlap). Lower effective electron mass typically also leads to a reduction in transparency carrier density. For simplicity, the analysis presented here will only focus on the design for GaAsSb-GaAsN laser structures.

One of the advantages of the "zig-zag" structure based on the tensile-strained GaAsN and compressively strained GaAsSb, compared with previous type-II designs at near $1.3-1.55 \mu \mathrm{m}$ [11] is the possibility of partial-strain compensation, allowing the growth of multiple "zig-zag" QW stages, to achieve the necessary optical gain in the laser device design. Another design advantage is the strong carrier confinement, both for the electrons and holes in their respective wells. The electrons are confined in the GaAsN electron-QWs, sandwiched with large $\Delta \mathrm{E}_{\mathrm{c}}$ of GaAs and GaAsSb. The holes in GaAsSb hole-QWs, are confined by the large $\Delta \mathrm{E}_{\mathrm{v}}$ of GaAsSb and $\operatorname{GaAsN}\left(\Delta \mathrm{E}_{\mathrm{v}} \sim 250-260 \mathrm{meV}\right)$. The "zig-zag" design of the GaAsN-GaAsSb QWs, has the potential for high-temperature and high-output-power laser operation as a result of the strong carrier confinement and the potential for reduced Auger recombination in the type-II structures [17].

In principle, the proposed design of the GaAsN-GaAsSb type-II QWs can be extended to longer wavelengths $(\lambda>2 \mu \mathrm{m})$. The resulting emission wavelength based on the calculated transitional energy levels including strain effects and the maximum quantum confinement effect (to illustrate the worst-case scenario) for the case of GaAsN-GaAsSb-GaAsN, with $40 \AA$ thickness for each individual layer, is shown in Fig. 2. The utilization of higher $\mathrm{N}$ and $\mathrm{Sb}$ content in the GaAsN or GaAsSb results in a smaller transition energy. A larger $\mathrm{N}$ content in the GaAsN will lower the electron-confined energy level. Likewise, larger Sb content in the GaAsSb, results in a higher confined-hole energy level. As shown in Fig. 2, the design utilizing $\mathrm{GaAs}_{0.965} \mathrm{~N}_{0.035}-\mathrm{GaAs}_{0.69} \mathrm{Sb}_{0.31}$, results in an emission wavelength up to $2 \mu \mathrm{m}$. The extension of the emission wavelength into the $3-4 \mu \mathrm{m}$ wavelength regions is also possible

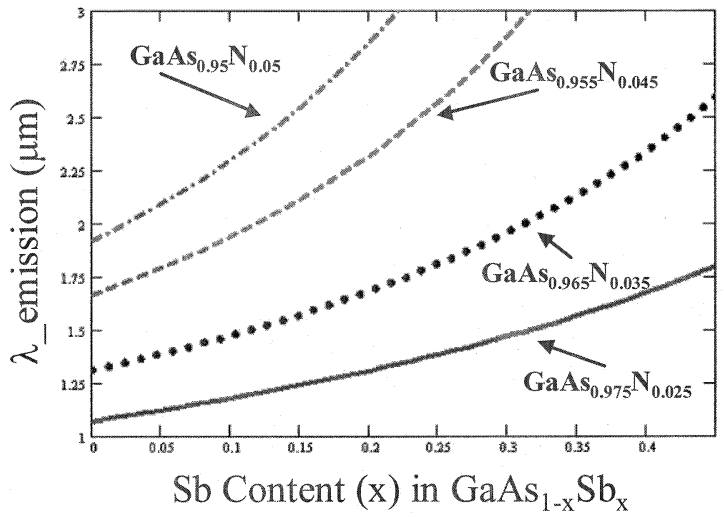

Fig. 2. Calculated emission wavelength of the GaAsN-GaAsSb-GaAs type-II QW with $40-\AA$ individual well thickness, for various $\mathrm{Sb}$ and $\mathrm{N}$ content, including the strain effect and maximum quantum confinement effect.

due to the strain-compensating effect of the GaAsN-GaAsSb structure. For example, structures utilizing $\mathrm{GaAs}_{0.955} \mathrm{~N}_{0.045}$ and $\mathrm{GaAs}_{0.69} \mathrm{Sb}_{0.31}$ result in emission wavelengths up to 3 $\mu \mathrm{m}$. In principle, the electron and hole confinement in the QWs should improve as the emission wavelength is extended due to the increased $\Delta \mathrm{E}_{\mathrm{c}}$ and $\Delta \mathrm{E}_{\mathrm{v}}$ for the higher $\mathrm{N}$ and Sb structures, although a penalty of reduced electron-hole wavefunctions overlap will occur at longer wavelengths.

\section{Design OPTIMIZATION FOR EMISSION WAVELENGTH AT $1500-\mathrm{nm}$}

In designing the "zig-zag" GaAsN-GaAsSb active region for $1.5-\mu \mathrm{m}$ wavelength emission, we try to choose the composition of GaAsN with minimum $\mathrm{N}$-content, and $\mathrm{GaAsSb}$ with a reasonable strain value. The $\mathrm{N}$-content for the GaAsN is chosen to be approximately $1.5 \%-2.5 \%$. The $\mathrm{Sb}$ content of the GaAsSb design ranges from $30 \%-40 \%$, which corresponds to $2.3 \%-3.0 \%$ compressive strain. The GaAsSb, with $30 \%-40 \%$ Sb content, should easily be realized without strain relaxation in the QW system, due to the thin dimension (25-35 $\AA$ ) of the GaAsSb and the strain compensating effect of the GaAsN-electron wells. The compositions of the GaAsN and GaAsSb are determined from the calculations of the transitional energy taking into account of the strain effect and the quantum confinement effect. The parameters for the $\mathrm{GaAsSb}$ and $\mathrm{GaAsN}$ material systems are taken following the treatments presented by Liu et al. [16] and Chow et al. [18], respectively. The quantum-confined energy levels are calculated by utilizing an effective mass approximation with a propagation matrix approach for multiple layer heterostructures [19]. By utilizing this method, normalized wavefunctions for both the electrons and holes are obtained. Obviously, a large overlap of the electron- and hole-wavefunctions is desirable for a large transitional matrix element and high material gain. Calculations utilizing a full (10-band $k \cdot p)$ bandstructure confirm reasonable agreement of the electron-hole wavefunction overlap values with the effective mass approximation results presented here [20].

In selecting the possible combinations of composition for the GaAsN and GaAsSb to achieve 1.5- $\mu \mathrm{m}$ emission wavelengths, 


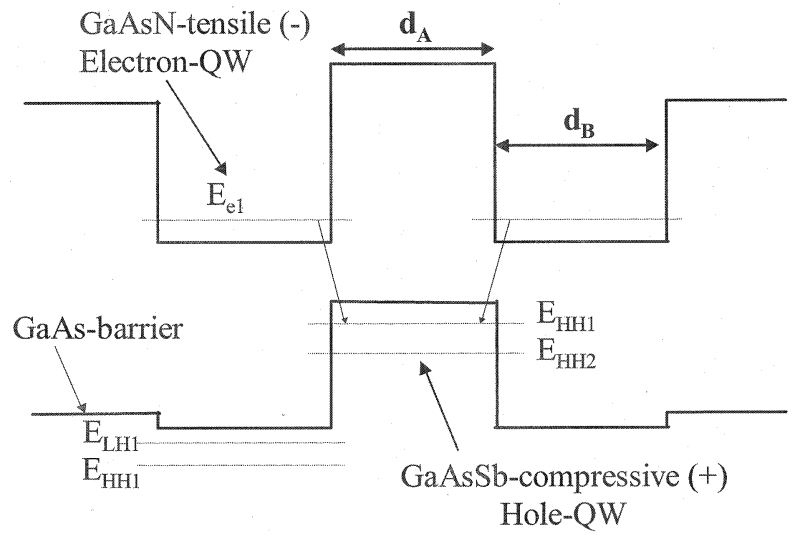

Fig. 3. Single-stage GaAsN-GaAsSb type-II QW laser, illustrating the respective hole energy band edge for GaAsN and GaAsSb.

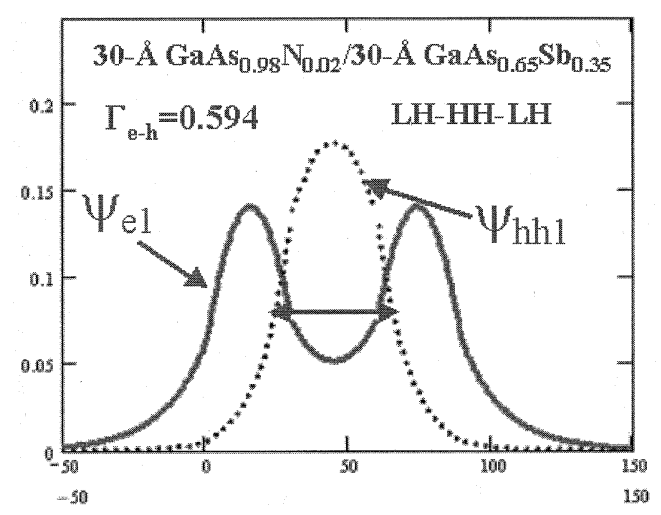

(a)

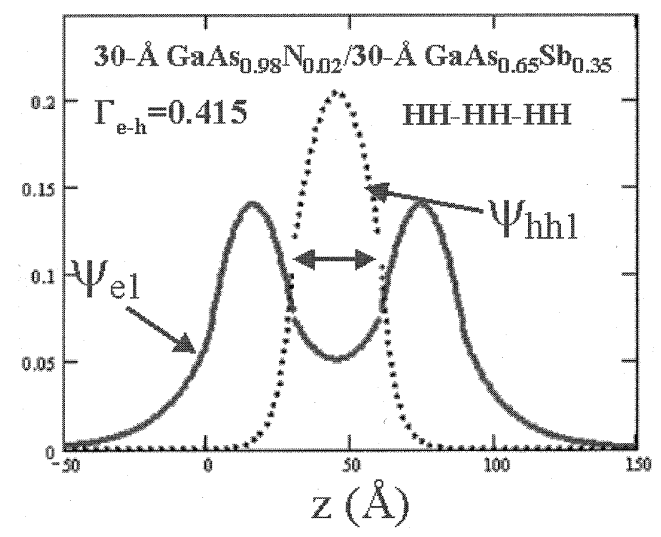

(b)

Fig. 4. Comparison of the hole-wavefunction and electron-hole wavefunction overlap for (a) light hole-heavy hole-light hole configuration and (b) the heavy hole/heavy hole/heavy hole configuration.

we consider first the "zig-zag" QW system with only a single stage, as shown in Fig. 3. The coupling of the wave-functions, primarily in the conduction bands (due to the smaller effective mass of the electrons) in multiple stages, results only in a very slight modification of the emission wavelength, as shown below.

Since the proposed type-II QW design consists of a compressively strain $\mathrm{GaAsSb}$ and a tensile-strain GaAsN, the lowest energy band edge for hole-confinement consists of heavy-hole in the GaAsSb well and light-hole in the GaAsN barriers. The light-hole barriers leads to an increased spreading of the hole

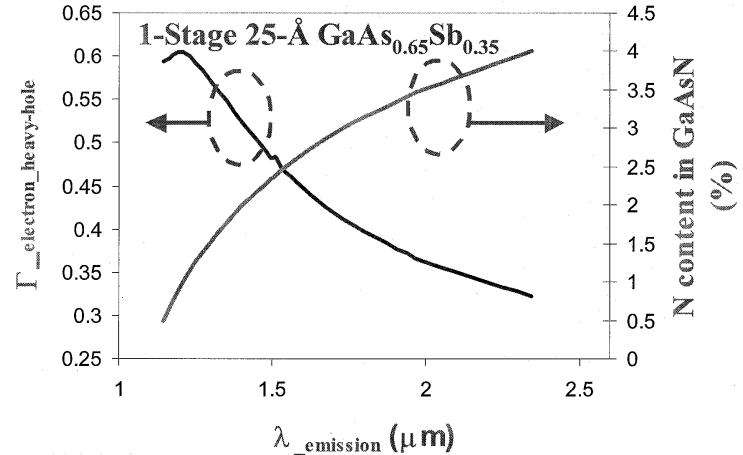

Fig. 5. Electron and hole wavefunctions overlap of a single stage GaAsN-GaAs $0.65 \mathrm{Sb}_{0.35}-\mathrm{GaAsN}$ QWs $(\mathrm{d}=25 \AA)$ as a function of $\lambda$, with its corresponding GaAsN composition.

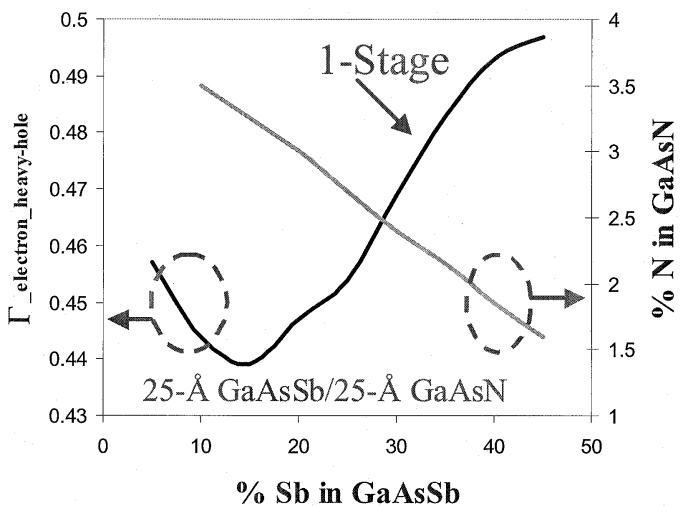

Fig. 6. Compositions of the GaAsN and GaAsSb and their respective electronand hole-wavefunction overlap for single (1-stage) QWs of $25 \AA / 25 \AA / 25 \AA$ of $\mathrm{GaAs}_{0.977} \mathrm{~N}_{0.023}-\mathrm{GaAs}_{0.65} \mathrm{Sb}_{0.35}-\mathrm{GaAs}_{0.977} \mathrm{~N}_{0.023} \mathrm{QW}$ systems for $\lambda=$ $1500 \mathrm{~nm}$

wavefunction, as shown in Fig. 4(a), ultimately improving the electron-hole wavefunction overlap. In reality, the hole-barrier consists of a mixture of heavy-hole and light-hole, leading to less spreading of the hole-wavefunction. The calculations presented here consider only a heavy-hole barrier in the GaAsN, as shown in Fig. 4(b), and thus represent a conservative estimate of the wavefunction spreading and electron-hole overlap.

Various compositions of the $\mathrm{GaAsN}$, with $\mathrm{GaAs}_{0.65} \mathrm{Sb}_{0.35}$, with individual thickness of $25 \AA$, as a function of the emission wavelength and the electron- and hole-wavefunction overlap $\left(\Gamma_{-\mathrm{e}-\mathrm{h}}\right)$ are shown in Fig. 5. The thickness of $25 \AA$ for the individual layers is taken arbitrarily as an example of the design space. Even though the $\Gamma_{-\mathrm{e}}$ hh tends to decrease as the emission wavelength is extended, this single stage "zig-zag" QW system, at $\lambda=1500 \mathrm{~nm}$, exhibits a high wavefunction overlap value as large as $48 \%$. These active regions can also be designed for the 1300-nm wavelength regime with even larger wavefunction overlap, as large as 58\%. Wavefunction overlaps above $37 \%$ are also predicted for an emission wavelength as long as $2 \mu \mathrm{m}$. However, the discussion here will only be limited to the optimization of the structure for $\lambda=1550 \mathrm{~nm}$.

The choice of the compositions of GaAsN and GaAsSb are critical in the selection of an optimized active region. As shown in Fig. 6, various compositions of the $25-\AA \mathrm{GaAsSb}$ and $25-\AA$ GaAsN QWs can be chosen, to achieve an emission wavelength 


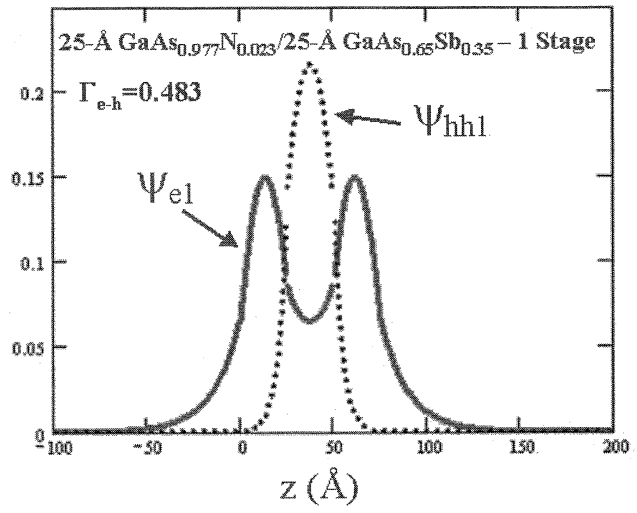

(a)

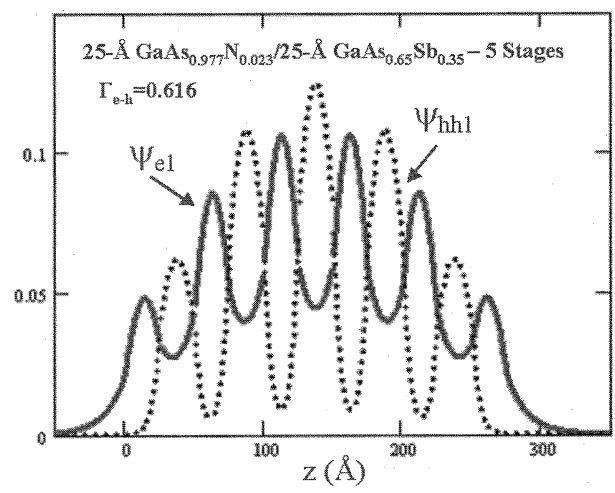

(b)

Fig. 7. Comparison of the hole-wavefunction and electron-hole wavefunction overlap for $1500-\mathrm{nm} \mathrm{GaAs}{ }_{0.977} \mathrm{~N}_{0.023}-\mathrm{GaAs}_{0.65} \mathrm{Sb}_{0.35}-\mathrm{GaAs}_{0.977} \mathrm{~N}_{0.023}$ of (a) single-stage QWs and (b) five-stage QWs.

at $1550 \mathrm{~nm}$. A large Sb-content GaAsSb composition will lead to minimal required $\mathrm{N}$ content to achieve the emission wavelength of $1.55 \mu \mathrm{m}$, which is important due to the reduced optical luminescence of higher-N content GaAsN materials. Although minimum $\mathrm{N}$-content is preferred, for high optical luminescence, the $\mathrm{N}$-content in the GaAsN should be large enough such that the electron energy level will have sufficiently deep barriers for strong electron confinement in the electron wells. A compromise between the $\mathrm{N}$ and $\mathrm{Sb}$ composition needs to be considered, by taking into account electron leakage suppression, strain of the combined QW system, and nonradiative recombination in GaAsN layers. Therefore, the optimal compositions of the layers will ultimately need to be experimentally determined. As shown in Fig. 6, the electron- and hole-wavefunction overlap values range from $44 \%$ to $50 \%$ for a single-stage QW system, with various compositions of GaAsSb and GaAsN for an emission wavelength of $1.5 \mu \mathrm{m}$. This shows that a large wavefunction overlap, with strong carrier confinement, can be achieved by selecting the proper composition of $\mathrm{Sb}$ and $\mathrm{N}$ in the design.

\section{Multiple “ZiG-ZAG” GaAsSB-GaAsN QW}

One of the challenges in designing the typical type-II QW systems is the low overlap between the electron wave-functions $\left(\psi_{\mathrm{c}}\right)$ and the hole wave-functions $\left(\psi_{\mathrm{h}}\right)$. The hole wave-functions of interest here are the heavy-hole wavefunctions $\left(\psi_{\mathrm{hh}}\right)$

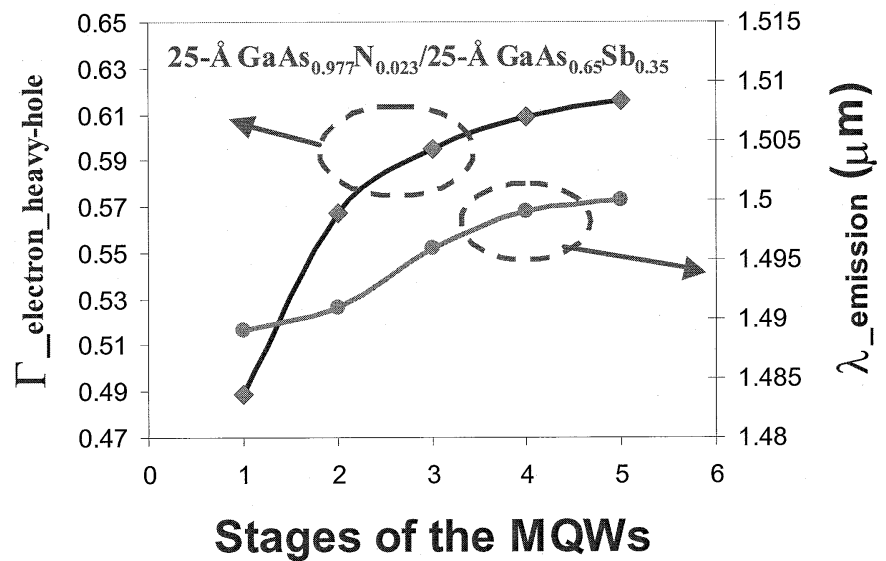

Fig. 8. Electron-hole wavefunction overlap of $\mathrm{GaAs}_{0.977} \mathrm{~N}_{0.023}-\mathrm{GaAs}_{0.65}$ $\mathrm{Sb}_{0.35}-\mathrm{GaAs}_{0.977} \mathrm{~N}_{0.023} \mathrm{Ws}$ as a function the number of stages, designed for $\lambda=1500-\mathrm{nm}$.

of the GaAsSb hole-QWs, since the transitions of the electron to heavy hole levels are favored due to the compressive strain of the GaAsSb hole-QW. To achieve a large wavefunction overlap in a MQW design, strong coupling for the electron wavefunction should be achieved such that the electron- and hole-wavefunctions exhibit a large amplitude in the GaAsSb region.

To achieve a strong coupling of the QWs, one can reduce the size of the QWs or implement a multiple-QW design. Reducing the size of the QWs is not preferable, due to other challenges, such as increased carrier leakage, reduced carrier capture efficiency, and difficulty in growth. Ideally, in the design of optimized structures, the QWs thickness is preferably above $25 \AA$ for strong carrier confinement and accurate thickness control in the MOCVD growth process.

By utilizing the self-strain-compensating mechanism of the GaAsN-GaAsSb "zig-zag” QW, large wavefunction overlaps can be achieved, due to the flexibility in implementing a multiple QW design. As shown in Fig. 7, both the electron- and hole-wavefunctions of the multiple QW system with five stages of $25-\AA \mathrm{GaAs}_{0.977} \mathrm{~N}_{0.023}-25-\AA \mathrm{GaAs}_{0.65} \mathrm{Sb}_{0.35}$ exhibits a significantly stronger coupling, which leads to a large wavefunction overlap value of $62 \%$, while preserving reasonable thick QWs $\left(\mathrm{d}_{\mathrm{A}}=\mathrm{d}_{\mathrm{B}}=25 \AA\right.$ ), as shown in Fig. 8. A $14 \%$ increase of the wavefunction overlap is predicted by utilizing the five-age QW system compared to that of the single-stage. From a practical perspective, only three QW stages are sufficient to achieve large overlap values above $60 \%$.

One potential disadvantage of the multiple "zig-zag" structure is the reduction of the peak optical gain, as a result of the decrease in the density of states [13]; thus, there is a design tradeoff to achieve the highest optical gain. A strong dispersion along the growth direction for electrons will reduce primarily the differential gain, although some reduction in the optical gain may also occur. This is due to the fact that the less-peaked density of states may partially or fully offset the increase in the electron-hole overlap. In GaSb-based type-II designs, multiple decoupled stages have been employed previously to achieve higher gain than a single stage by maintaining a two-dimensional energy dispersion relationship [13]. 


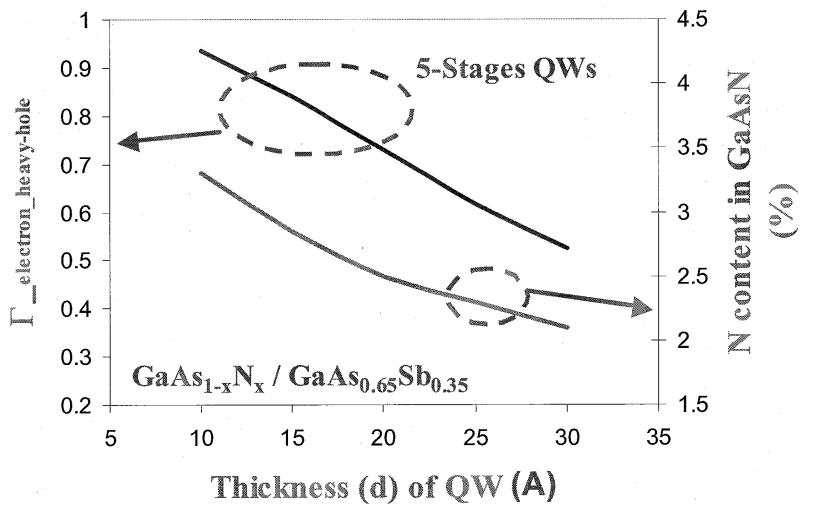

Fig. 9. $\Gamma_{\text {e_hole }}$ of $\mathrm{GaAs}_{1-x} \mathrm{~N}_{x}-\mathrm{GaAs}_{0.65} \mathrm{Sb}_{0.35}-\mathrm{GaAs}_{1-x} \mathrm{~N}_{x}$ QWs for thickness $\left(\mathrm{d}=\mathrm{d}_{\mathrm{A}}=\mathrm{d}_{\mathrm{B}}\right)$ of QWs, with five-stage QWs and designed for $\lambda=1500 \mathrm{~nm}$.

The other technique to improve the coupling of the wavefunctions is to reduce the thickness of the individual layers of GaAsN or GaAsSb. In principle, one can vary the thickness of the individual layers independent of one another such that high coupling of the wavefunctions can be achieved. Calculations of the wavefunction overlap for various QW thickness, with $\mathrm{d}_{\mathrm{A}}=\mathrm{d}_{\mathrm{B}}$ at $\lambda=1500 \mathrm{~nm}$, are shown in Fig. 9. From the calculation, very thin QW systems of 15-20 A result in very large wavefunction overlap up to $84 \%$ and $74 \%$, respectively, for the five-stage QW design.

\section{SUMMARY}

We propose a novel alternative active region based on the type-II transition of (In)GaAsN-GaAsSb, with an emission wavelength ranging from 1.2 to $3 \mu \mathrm{m}$. The proposed (In)GaAsN-GaAsSb QW active region holds for achieving the high-performance $\lambda=1.5 \mu \mathrm{m}$ GaAs-based edge-emitting lasers and VCSELs. The unique feature of the proposed design is that it allows for strong carrier confinement (for low device temperature sensitivity), large overlap of the electron-hole wavefunctions (for high optical gain), and the ability to implement strain compensation (for MQW active designs). An optimum design indicates that $62 \%$ electron-hole wavefunction overlap, for the $\lambda=1500-\mathrm{nm}$ wavelength regime, is feasible by utilizing a self-strain compensating strongly coupled MQW system with a reasonable well thickness, $\mathrm{d}_{\mathrm{A}}=\mathrm{d}_{\mathrm{B}}=25 \AA$.

Although the analysis presented here is limited to the case for GaAsSb-GaAsN for 1500-nm lasers, the structure can be easily modified to the GaAsSb-InGaAsN type-II QWs structures if necessary. The potential advantages of the InGaAsN electron-QW is the smaller effective electron mass for the InGaAsN QW in comparison to that of GaAsN QW. Lower effective electron mass typically leads to a reduction in the transparency carrier density. The utilization of low In-content InGaAsN QWs will allow the flexibility of engineering the QWs to be either tensile strained, lattice matched, or compressive strained.

\section{ACKNOWLEDGMENT}

The authors would like to acknowledge helpful technical discussions and comments from Dr. J. R. Meyer and Dr. I. Vurgaftman, both of Naval Research Laboratory, Washington, DC.

\section{REFERENCES}

[1] D. I. Babic, K. Streubel, R. P. Mirin, N. M. Margalit, J. E. Bowers, E. L. Hu, D. E. Mars, Y. Long, and K. Carey, "Room-temperature continuous-wave operation of 1.54- $\mu \mathrm{m}$ vertical-cavity lasers," IEEE Photon. Technol. Lett., vol. 7, pp. 1225-1227, Nov. 1995.

[2] J. Boucart, C. Starck, F. Gaborit, A. Plais, N. Bouche, E. Derouin, L. Golstein, C. Fortin, D. Carpentier, P. Salet, F. Brillouet, and J. Jacquet, "1-mW CW-RT monolithic VCSEL at $1.55 \mu \mathrm{m}$," IEEE Photon. Technol. Lett., vol. 11, pp. 629-631, June 1999.

[3] E. Hall, S. Nakagawa, G. Almuneau, J. K. Kim, and L. A. Coldren, "Room-temperature, $\mathrm{CW}$ operation of lattice-matched long-wavelength VCSELs," Electron. Lett., vol. 36, no. 17, pp. 1465-1467, 2000.

[4] R. Shau, M. Ortsiefer, J. Rosskopf, G. Bohm, F. Kohler, and M. C. Amann, "Vertical-cavity surface-emitting laser diodes at $1.55 \mu \mathrm{m}$ with large output power and high operation temperature," Electron. Lett., vol. 37, no. 21, pp. 1295-1296, 2001.

[5] C. K. Lin, D. P. Bour, J. Zhu, W. Perez, M. H. Leary, A. Tandon, S. W. Corzine, and M. R. Tan, "High temperature continuous-wave operation of 1.3-1.55 $\mu \mathrm{m}$ VCSELs with InP/Air-GaP DBRs," in Proc. IEEE Int. Semiconductor Lasers Conf. 2002, Garmisch-Partenkirchen, Germany, Oct. 2002, Paper ThA6.

[6] A. F. Phillips, A. F. Sweeney, A. R. Adams, and P. J. A. Thijs, "Temperature dependence of 1.3- and 1.5- $\mu \mathrm{m}$ compressively strained $\operatorname{InGaAs}(\mathrm{P})$ MQW semiconductor lasers," IEEE. J. Select. Topics Quantum Electron., vol. 5, pp. 401-412, May/June 1999.

[7] N. Tansu and L. J. Mawst, "Low-threshold strain-compensated In$\operatorname{GaAs}(\mathrm{N})(\lambda=1.19-1.31 \mu \mathrm{m})$ quantum well lasers," IEEE Photon. Technol. Lett., vol. 14, pp. 444-446, Apr. 2002.

[8] N. Tansu, N. J. Kirsch, and L. J. Mawst, "Low-threshold-current-density 1300-nm dilute-nitride quantum well lasers," Appl. Phys. Lett., vol. 81, no. 14, pp. 2523-2525, Sept. 2002.

[9] N. Tansu, J. Y. Yeh, and L. J. Mawst, "Realization of low-threshold 1382-nm InGaAsN quantum well lasers by metalorganic chemical vapor deposition," Appl. Phys. Lett., submitted for publication.

[10] M. O. Fischer, M. Reinhardt, and A. Forchel, "Room-temperature operation of GaInAsN-GaAs laser diodes in the 1.5- $\mu \mathrm{m}$ range," IEEE J. Select. Topics Quantum Electron., vol. 7, pp. 149-151, Mar--Apr. 2001.

[11] W. Ha, V. Gambin, M. Wistey, S. Bank, S. Kim, and J. S. Harris, Jr., "Long-wavelength GaInNAs(Sb) lasers on GaAs," IEEE J. Quantum Electron., vol. 38, pp. 1260-1267, Sept. 2002.

[12] P. Dowd, W. Braun, D. J. Smith, C. M. Ryu, C.-Z. Guo, S. L. Chen, U. Koelle, S. R. Johnson, and Y.-H. Zhang, "Long wavelength (1.3 and 1.5 $\mu \mathrm{m})$ photoluminescence from InGaAs/GaPAsSb quantum wells grown on GaAs," Appl. Phys. Lett., vol. 75, no. 9, pp. 1267-1269, 1999.

[13] J. R. Meyer, C. A. Hoffman, F. J. Bartoli, and L. R. Ram-Mohan, "Type II-quantum well lasers for the mid-wavelength infrared," Appl. Phys. Lett., vol. 67, no. 6, pp. 757-759, 1995.

[14] J. Wu, W. Shan, W. Walukiewicz, K. M. Yu, J. W. Ager, III, E. E. Haller, H. P. Xin, and C. W. Tu, "Effect of band anticrossing on the optical transition in $\mathrm{GaAs}_{1-x} \mathrm{~N}_{x} / \mathrm{GaAs}$ multiple quantum wells," Phys. Rev. $B$, vol. 64, p. 85320 , August 2001.

[15] C. G. Van de Walle, "Band lineups and deformation potentials in the model-solid theory," Phys. Rev. B, vol. 39, pp. 1871-1883, 1989.

[16] G. Liu and S.-L. Chuang, "Optical gain of strained GaAsSb/GaAs quantum-well lasers: A self consistent approach," J. Appl. Phys., vol. 88 , no. 10 , pp. 5554-5561, Nov. 2000.

[17] J. R. Meyer, C. L. Felix, W. W. Bewley, I. Vurgaftman, E. H. Aifer, L. J. Olafsen, J. R. Lindle, C. A. Hoffman, M. J. Yang, B. R. Bennett, B. V. Shanabrook, H. Lee, C. H. Lin, S. S. Pei, and R. H. Miles, "Auger coefficient in type-II InAs/ $\mathrm{Ga}_{1-x} \mathrm{In}_{x} \mathrm{Sb}$ quantum wells," Appl. Phys. Lett., vol. 73, no. 20, pp. 2857-2859, 1998.

[18] W. W. Chow, E. D. Jones, N. A. Modine, A. A. Allerman, and S. R. Kurtz, "Laser gain and threshold properties in compressive-strained and lattice-matched GaInNAs/GaAs quantum wells," Appl. Phys. Lett., vol. 75, pp. 2891-2893, 1999.

[19] S. L. Chuang, Physics of Optoelectronic Devices. New York: Wiley, 1995.

[20] I. Vurgaftman, J. R. Meyer, N. Tansu, and L. J. Mawst, "(In)GaAsN-GaAsSb Type-II "W" Quantum-Well Lasers for Emission at $\lambda=1.55 \mu \mathrm{m}$," Appl. Phys. Lett., vol. 83, no. 14, Oct. 2003. 
Nelson Tansu was born in Medan, North Sumatra, Indonesia, in 1977. He received the B.S. degree in applied mathematics, electrical engineering, and physics (AMEP) in 1998 and the Ph.D. degree in electrical engineering in 2003, both from the University of Wisconsin-Madison.

Since July 2003, he has been an Assistant Professor in the Department of Electrical and Computer Engineering, P. C. Rossin College of Engineering and Applied Science, Lehigh University, Bethlehem, PA. He has also been a Faculty Member in the Center for Optical Technologies, Lehigh University, since July 2003. His research work has included the design, fabrication, and MOCVD growth of novel-active material GaAs-based vertical cavity lasers (VCSELs) for all practical transmission windows of optical-communications systems, $850-\mathrm{nm}$ VCSELs (InGaAsP QW), 1300-nm (InGaAsN QW) and 1550-nm diode lasers, the physics of semiconductor quantum-well lasers encompassing recombination mechanisms, optical gain, carrier transport, and temperature characteristics. His research interests are in the areas of photonics, optoelectronics, nanotechnology, and photonic crystals. He has published widely in numerous refereed international journal and conference publications and he currently holds several U.S. patents. He has also given numerous lectures, seminars, and invited talks in universities, research institutions, and conferences in the U.S., Canada, Europe, and Asia.

Dr. Tansu was a recipient of the Bohn Scholarship, the WARF Graduate University Fellowship, the Vilas Graduate University Fellowship, and the Graduate Dissertator Travel Funding Award at the University of Wisconsin-Madison. He also received the 2003 Harold A. Peterson ECE Best Research Paper Award (First Prize) from the University of Wisconsin-Madison.
Luke J. Mawst (M'88-SM'93) was born in Chicago, IL, in 1959. He received the B.S. degree in engineering physics and the M.S. and Ph.D. degrees in electrical engineering from the University of Illinois at Urbana-Champaign in 1982, 1984, and 1987, respectively. His dissertation research involved the development of index-guided semiconductor lasers and laser arrays grown by MOCVD.

He joined TRW, Inc., Redondo Beach, CA, in 1987, where he was a Senior Scientist in the Research Center, engaged in design and development of semiconductor lasers using MOCVD crystal growth. He is currently an Associate Professor at the University of Wisconsin-Madison, where he is involved in the development novel III/V compound semiconductor device structures, including vertical-cavity surface-emitting lasers (VCSELs), active photonic lattice structures, InGaAsN lasers, and high-power Al-free diode lasers. His previous work has included developing a novel single-laser structure, the ARROW laser, as a source for coupling high powers into fibers, the development of two-dimensional coherent surface-emitting arrays, vertical-cavity surface emitters, and distributed-feedback laser structures. He has authored or coauthored more than 120 technical papers and holds 15 patents.

Dr. Mawst received the TRW Group Level Chairman's Award as co-inventor of the Resonant Optical Waveguide (ROW) antiguided array and for contributions to its development as a practical source of high coherent power. 